\title{
Toxicité rénale des immunoglobulines intraveineuses
}

\section{Intravenous Immunoglobulin Nephrotoxicity}

\author{
Y. Luque \\ Reçu le 1 avril 2018; accepté le 27 juin 2018 \\ (C) SRLF et Lavoisier SAS 2018
}

Résumé Les immunoglobulines intraveineuses (IgIV) sont largement prescrites de nos jours à visée substitutive ou à visée immunomodulatrice. Les IgIV sont prescrites à faible dose (environ $200 \mathrm{mg} / \mathrm{kg}$ ) dans les déficits immunitaires afin de substituer les IgIV endogènes manquantes ou à forte dose (1 à $2 \mathrm{~g} / \mathrm{kg}$ et par cure) à visée immunomodulatrice dans diverses pathologies inflammatoires ou auto-immunes. Ces produits, fabriqués à partir de plasmas de donneurs sains, peuvent s'accompagner d'une toxicité rénale décrite dès les années 1990. Il s'agit d'une toxicité tubulaire pouvant aboutir à des insuffisances rénales aiguës sévères. L'analyse des biopsies rénales révèle des vacuolisations des cytoplasmes tubulaires et des lésions de nécrose tubulaire aiguë. Les excipients glucidiques et en particulier le saccharose sont les principaux facteurs favorisant cette néphrotoxicité. L'utilisation d'IgIV sans saccharose a permis de diminuer nettement les cas d'insuffisance rénale aiguë et doit être préférée chez les patients ayant une insuffisance rénale préalable ou à risque d'en développer (patients âgés, diabétiques, obèses, hypovolémiques ou transplantés rénaux).

Mots clés IgIV · Néphrotoxicité · Saccharose · Insuffisance rénale aiguë · Tubulopathie

\begin{abstract}
Intravenous immunoglobulin (IVIG) indications have been dramatically increased recently in many medical fields as antibody replacement therapy at low-dose or for immunomodulatory purposes at high-dose $(1-2 \mathrm{~g} / \mathrm{kg})$. IVIG contains the pooled immunoglobulins from the plasma of approximately a thousand or more blood donors and its nephrotoxicity has been described since the 1990s and mainly attributed to sucrose stabilizers. Histological analysis of the kidneys showed osmotic nephrosis lesions with tubular vacuolization and acute tubular necrosis. From the mid-
\end{abstract}

\section{Y. Luque $(\square)$}

Urgences néphrologiques et transplantation rénale, hôpital Tenon, Assistance Publique - Hôpitaux de Paris, Unité de Recherche Inserm UMR_S1155, Sorbonne Université 4, rue de la Chine, F-75020, Paris, France

e-mail : yosu.luque@aphp.fr 2000s forward, some new IVIG formulations without sucrose have been used to decrease acute kidney injury (AKI) incidence. These formulations should be preferred in patients at risk of AKI including old patients, diabetes mellitus, obese, hypovolemic or with a chronic kidney disease or a renal graft.

Keywords IVIG $\cdot$ Nephrotoxicity $\cdot$ Sucrose $\cdot$ Acute kidney injury $\cdot$ Tubulopathy

Les immunoglobulines humaines polyvalentes intraveineuses (IgIV) sont utilisées dans de nombreux domaines de la médecine actuelle à visée substitutive ou immunomodulatrice et ont vu leurs indications multipliées ces dernières années. Les trois quarts des IgIV produites sont utilisées entre les États-Unis et l'Europe, et la consommation mondiale est passée de $10 \mathrm{t}$ en 1992 à quasiment $100 \mathrm{t}$ en 2010 (source : The WorldWide Plasma Fractions Market). En France, la consommation de ce produit a augmenté de $12 \%$ par an entre 2004 et 2010, avec quasiment 6 t d'IgIV utilisées en 2010 (source : Groupement pour l'élaboration et la réalisation de statistiques [GERS]). Les indications de ce produit s'élargissant de nos jours, ce volume de consommation pourrait encore s'accroître les années à venir. Ce sont des produits coûteux avec un prix moyen de $40 €$ par gramme d'IgIV en France (source : HAS, 2008). Utilisées à grande échelle, les IgIV ont un potentiel néphrotoxique variable en fonction de la formulation utilisée qui est importante à connaître pour les prescripteurs.

Les IgIV ont été utilisées à partir des années 1960 comme traitement substitutif pour les patients ayant des déficits immunitaires, mais la technique de purification, imparfaite à cette époque, avait comme conséquence des réactions anaphylactiques fréquentes. L'utilisation des IgIV a été élargie dans cette indication substitutive au cours des années 1980, avec des produits très purifiés et mieux tolérés [1]. Cependant, à partir des années 1990, les indications de ces produits se sont multipliées, car on rapporte une action immunomodulatrice dans le purpura thrombopénique immunologique (PTI) [2], le syndrome de Guillain-Barré ou la maladie de Kawasaki [3,4]. 
Les IgIV, produites à partir de plasmas humains d'un pool de donneurs (au moins 1000 donneurs d'après les critères de l'OMS), contiennent principalement des IgG de types 1 et 2 .

Les immunoglobulines sont extraites du plasma puis purifiées par différentes techniques afin de sélectionner les immunoglobulines G. Par ailleurs, on inactive les éventuels pathogènes pour assurer un produit ne comportant pas de risque infectieux. Le produit final diffère par la composition en types d'immunoglobulines et par les excipients (Tableau 1).

En effet, toutes les IgIV contiennent plus de $90 \%$ d'immunoglobulines, des stabilisants et excipients qui peuvent être des sucres (glucose, maltose, saccharose), des acides aminés (glycine, proline) et du sodium. Malgré la purification ou de façon intentionnelle, une faible teneur en IgA persiste dans certains de ces produits. Celle-ci est importante chez les patients ayant un déficit en IgA, qui parfois peuvent développer des anticorps anti-IgA induisant des réactions anaphylactiques lors de la perfusion de ces IgIV. Les sucres contenus comme excipients ont aussi une grande importance, car le saccharose peut entraîner des lésions de néphrose osmotique, le maltose altère la mesure des lecteurs glycémiques, et le glucose peut déséquilibrer la glycémie chez les patients diabétiques. Enfin, la teneur en sodium est à prendre en compte chez les patients en surcharge hydrosodée ou insuffisants cardiaques ou rénaux.

La dose d'IgIV administrée est différente en fonction de l'indication. En effet, pour substituer les déficits immunitaires, on utilise des faibles doses qui commencent à 300 $400 \mathrm{mg} / \mathrm{kg}$ par mois et vont jusqu'à $900 \mathrm{mg} / \mathrm{kg}$ par mois. C'est dans ce cadre que les IgIV sous-cutanées ont pris leur place. Dans l'indication immunomodulatrice, les doses sont plus élevées de l'ordre de 1 à $2 \mathrm{~g} / \mathrm{kg}$ toutes les trois à quatre semaines, mais ces dosages relèvent souvent d'une approche empirique. La demi-vie des IgIV est d'environ trois semaines, et elle dépend de leur concentration plasmatique et du métabolisme des patients.

Une autre donnée intéressante à signaler est que, dans $50 \%$ des cas, les IgIV sont utilisées de nos jours dans des

Tableau 1 Excipients des immunoglobulines intraveineuses disponibles en France

\begin{tabular}{|c|c|c|c|}
\hline Produit & Laboratoire & $\begin{array}{l}\text { Excipient } \\
\text { glucidique }\end{array}$ & $\begin{array}{l}\text { Autre } \\
\text { excipient }\end{array}$ \\
\hline Tegeline $^{\circledR}$ & LFB & Saccharose & \\
\hline Sandoglobuline $\left.{ }^{(}\right)$ & CSL Behring & Saccharose & \\
\hline Gammagard $^{\circledR}$ & Baxter & Glucose & $\begin{array}{l}\text { Glycine } \\
\text { PEG } 2\end{array}$ \\
\hline Octagam $^{\circledR}$ & Octapharma & Maltose & \\
\hline Privigen ${ }^{\circledR}$ & CSL Behring & Aucun & L-proline \\
\hline Kiovig $^{\circledR}$ & Baxter & Aucun & Glycine \\
\hline Clairyg $^{\circledR}$ & LFB & Mannitol & Glycine \\
\hline
\end{tabular}

indications qui ne sont pas celles de l'autorisation de mise sur le marché (AMM) en Europe comme aux États-Unis [5].

En effet, une utilisation empirique et récente de ces molécules dans son indication immunomodulatrice et le faible nombre d'études puissantes étudiant leur efficacité pourraient expliquer ce phénomène.

\section{Mécanismes d'action des IgIV}

Le mécanisme d'action des IgIV n'est pas complètement élucidé de nos jours, mais de notables avancées se sont produites ces dernières années (Fig. 1). Dans les déficits immunitaires congénitaux, les IgIV sont administrées à visée substitutive de façon itérative afin de rétablir un taux d'anticorps circulants suffisant pour restituer une immunité humorale permettant de diminuer le taux d'infections [6]. Elles sont aussi utilisées plus rarement dans des déficits immunitaires secondaires liés à la leucémie lymphoïde chronique [7], à l'allogreffe de cellules souches hématopoïétiques, à l'infection chronique par le parvovirus B19 ou le VIH [8].

La deuxième utilisation des IgIV est à visée immunomodulatrice dans des maladies inflammatoires liées ou non à un phénomène d'auto-immunité. Malgré une utilisation très fréquente des IgIV dans cette indication immunomodulatrice qui concerne le PTI, le syndrome de Guillain-Barré ou la maladie de Kawasaki par exemple, l'action des immunoglobulines humaines polyvalentes à forte dose sur la réponse inflammatoire n'est pas encore complètement élucidée. L'utilisation empirique des IgIV dans ces maladies inflammatoires impliquant ou non des autoanticorps a précédé la compréhension de leur mécanisme d'action. Le rôle immunomodulateur des IgG a été suggéré par l'incidence accrue de maladies auto-immunes chez des enfants ayant

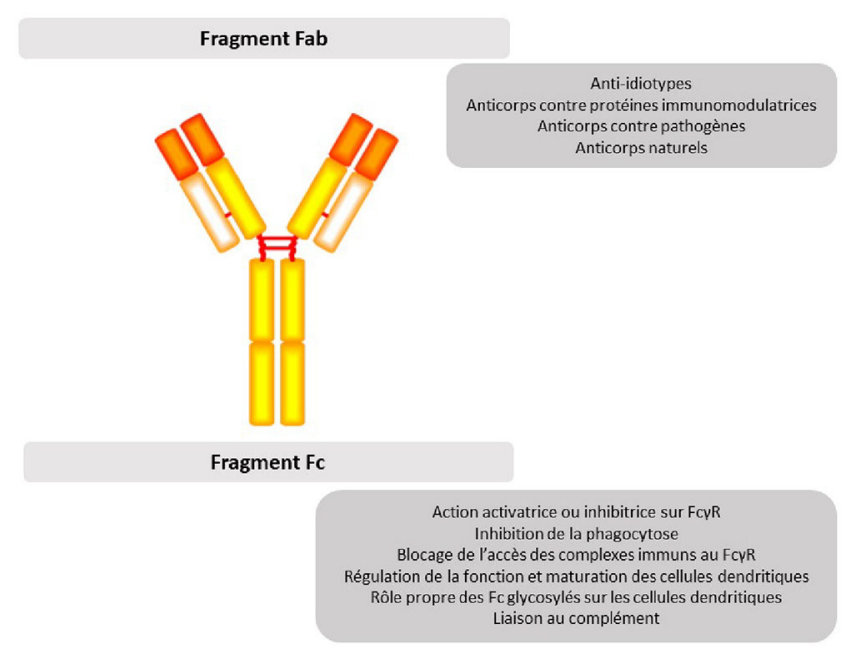

Fig. 1 Mécanismes d'action proposés des immunoglobulines intraveineuses 
une hypogammaglobulinémie [2]. Ces dernières années, de grands progrès ont été réalisés dans la compréhension physiopathologique de ce phénomène grâce à des études in vitro et sur des modèles murins de PTI, d'arthrite inflammatoire ou de néphrite toxique [9]. Différentes études ont apporté des preuves sur le rôle immunomodulateur des fragments $\mathrm{Fab}$ mais surtout du fragment Fc des immunoglobulines.

L'action des fragments Fab impliquerait le mécanisme antigène-anticorps dans l'immunorégulation liée aux IgIV. En effet, certaines immunoglobulines des préparations, issues de centaines de donneurs humains, seraient dirigées contre des cytokines pro-inflammatoires [10] comme BAFF (B-cell activating factor, une cytokine de la famille du facteur de nécrose tumorale), des récepteurs membranaires comme le CD95 (fas) [11,12], des molécules d'adhésion ou contre les anticorps pathogènes eux-mêmes. Même si des études in vitro soutiennent cette théorie, les études in vivo ont des difficultés à démontrer un rôle principal de ce mécanisme lié aux fragments Fab probablement à cause du faible taux d'anticorps spécifiques contenus dans les préparations commerciales d'IgIV.

En revanche, de nombreuses études in vitro et in vivo ont montré un rôle immunomodulateur principal au fragment $\mathrm{Fc}$ des immunoglobulines G. En effet, l'injection de fragments Fc purifiés chez des enfants ayant un PTI a montré leur efficacité dans une étude publiée en 1993 [13]. Le fragment $\mathrm{Fc}$ agirait sur les récepteurs $\mathrm{Fc} \gamma \mathrm{R}$, protéines présentes à la surface de certaines cellules - dont les lymphocytes B, les cellules dendritiques folliculaires, les lymphocytes NK, les macrophages, les neutrophiles, les éosinophiles, les basophiles, les thrombocytes et les mastocytes - qui contribuent aux fonctions protectrices du système immunitaire [14]. En physiologie, il existe une compétition entre des récepteurs aux Fc activateurs et inhibiteurs chez l'homme. Plusieurs études montrent que les IgG activent des récepteurs FcyRIIB inhibiteurs situés dans les cellules phagocytaires de l'immunité innée et inhibent des récepteurs $\mathrm{Fc}$ activateurs comme le FcyRIII chez la souris [14]. L'injection d'IgIV aboutirait à une surexpression du récepteur FcyRIIB chez l'homme [15]. Par ailleurs, le fragment Fc stimulerait aussi la prolifération des lymphocytes $T$ régulateurs [16] et aurait une action inhibitrice sur les lymphocytes B [17]. Enfin, des études plus récentes accordent un rôle important aux immunoglobulines ayant des résidus glycosylés [18]. Celles-ci agiraient sur des récepteurs membranaires des cellules immunitaires reconnaissant de façon spécifique des fragments Fc glycosylés. Ces récepteurs spécifiques auraient un rôle principal dans l'action anti-inflammatoire des IgIV [19]. La compréhension du mécanisme d'action des IgIV a donc avancé ces dernières années et accorde un rôle principal immunomodulateur à l'interaction du fragment $\mathrm{Fc}$ des immunoglobulines avec les récepteurs situés à la surface des cellules immunitaires. Des études chez l'homme seront nécessaires afin de préciser le mécanisme d'action et l'importance du rôle de la glycosylation des immunoglobulines.

\section{Effets indésirables des IgIV et risque de néphrotoxicité}

La plupart des effets indésirables des IgIV sont mineurs et transitoires comme les céphalées, les frissons ou les flushs. Cependant, des effets secondaires graves peuvent survenir chez 2 à $6 \%$ des patients. Ceux-ci incluent les insuffisances rénales aiguës, les thromboses veineuses, l'hémolyse et les réactions anaphylactiques. Ces réactions peuvent survenir dans un délai immédiat (anaphylaxie, céphalées) ou de façon différée. On notera les réactions anaphylactiques chez les patients n'ayant pas de taux détectable d'IgA qui ont développé des anticorps anti-IgA. Comme on l'a écrit précédemment, certaines IgIV peuvent contenir des taux variables d'IgA qui peuvent entraîner des réactions anaphylactiques chez ces patients [20]. Par ailleurs, on retrouve dans la littérature médicale des cas d'hémolyse intravasculaire liée à des anticorps antiérythrocytaires contenus dans les IgIV [21]. Ces cas sont notés avec des doses élevées d'IgIV de l'ordre de $2 \mathrm{~g} / \mathrm{kg}$. Dans une étude, on note aussi une neutropénie transitoire chez 16 patients [22]. Enfin, des études in vitro et in vivo prouvent que les IgIV augmentent la viscosité sanguine et peuvent entraîner des thromboses veineuses à partir du point de ponction $[23,24]$. Des événements cardiovasculaires coronaires et des AVC ont aussi été rapportés [25]. Ces effets sont prévenus par une bonne hydratation, de faibles débits de perfusion et par le choix d'une préparation avec une faible osmolarité.

Les indications des IgIV se multiplient y compris en transplantation rénale, bien que ces produits comportent un risque de néphrotoxicité qui s'expliquerait par un mécanisme de néphrose osmotique [26]. Le premier cas d'insuffisance rénale aiguë (IRA) liée aux IgIV a été rapporté en 1987 [27]. L'utilisation des IgIV s'est étendue dans les années 1980-1990, et on dénombre un grand nombre d'articles datant des années 1990 rapportant des insuffisances rénales modérées et transitoires après la perfusion de ces produits [28-32]. L'incidence de l'IRA liée aux IgIV a été estimée à $7 \%$ chez des patients à fonction rénale normale traités avec de fortes doses $(1-2 \mathrm{~g} / \mathrm{kg})$ pour des maladies inflammatoires ou infectieuses [33], puis à environ $1 \%$ par des études plus récentes [34]. Une excellente revue récente des effets des excipients des IgIV sur la fonction rénale détaille la fréquence de survenue de l'IRA, avec une fréquence déclarative inférieure à $1 \%$ pour les produits sans saccharose [35]. L'insuffisance rénale liée aux IgIV est de survenue rapide dans les deux à trois jours suivant le début de la perfusion avec un tableau d'atteinte tubulaire. Elle est souvent réversible à distance, et des cas exceptionnels 
décrits lors de l'utilisation des spécialités avec saccharose ont eu la nécessité de recourir à l'épuration extrarénale. L'étude de Brannagan et al. de 1996 [36] qui étudiait la fonction rénale chez des patients neurologiques recevant des perfusions d'IgIV n'a reporté qu'un seul cas d'IRA. Dans l'étude de Sati et al. en 2001, on dénombre 4 patients sur $55(7 \%)$ qui développent une IRA avec des spécialités contenant du saccharose et dans des indications hématologiques [33]. On dénombre un certain nombre de cas exceptionnels d'IRA sévère avec nécessité d'épuration extrarénale. Dans les études datant des années 1990, une épuration extrarénale était nécessaire dans environ $40 \%$ des cas d'IRA liée aux IgIV [37]. Entre 1985 et 1998, la FDA a comptabilisé 54 cas d'IRA après perfusion d'IgIV [37]. L'analyse histologique des reins montrait des lésions de néphrose osmotique chez la quasi-totalité des patients. Tous les cas sauf un étaient liés à des préparations contenant du saccharose. Le saccharose a donc été incriminé dans la néphrotoxicité des IgIV, car on a mis en relation ces lésions avec les cas de néphropathie au saccharose publiés dans les années 1930 [38]. Ce produit était utilisé à l'époque afin de diminuer la pression intracrânienne dans l'œdème cérébral. La néphropathie liée aux IgIV a alors été assimilée à la néphropathie liée à la perfusion de saccharose (sucrose en anglais) $[38,39]$. En effet, dans les deux cas, à l'examen histologique du rein, on trouve des lésions de nécrose tubulaire aiguë avec des vacuolisations des cellules tubulaires [40].

La description histologique de la néphrose osmotique (Fig. 2) a débuté dans les années 1930 avec les IRA liées aux perfusions de saccharose. Dans les modèles murins et chez l'homme, on note une dégénération vacuolaire des cellules tubulaires proximales. Des lésions de nécrose tubulaire

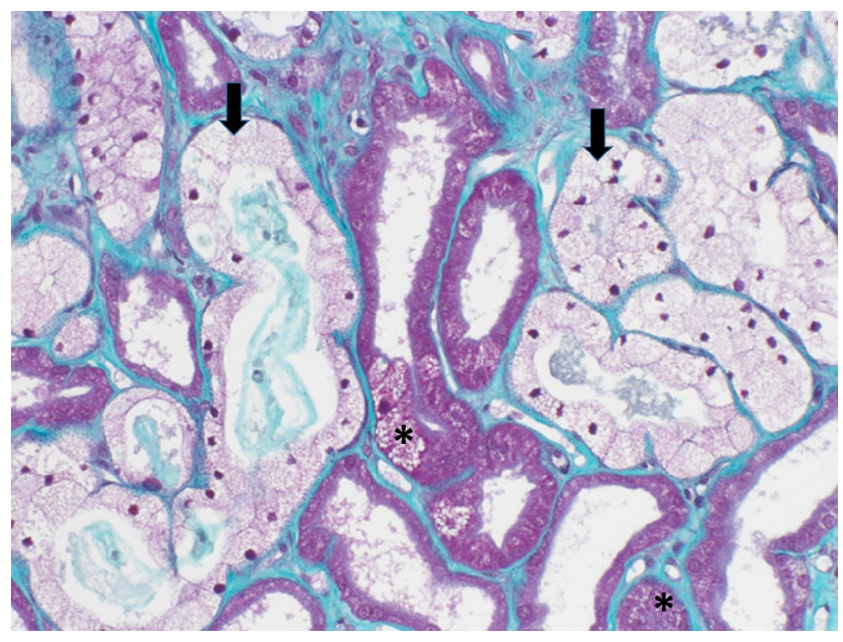

Fig. 2 Biopsie rénale, microscopie optique : micro- $(*)$ et macrovacuoles $(\rightarrow)$ dans les cellules tubulaires proximales et nécroses tubulaires caractéristiques de la néphrose osmotique aiguë sont aussi notées. Les lésions initiales sont composées de microvacuoles apicales qui grandissent en taille au fur et à mesure de l'agression et confluent pour aboutir à des macrovacuoles plus tardivement. Ces vacuoles sont identifiées par histochimie en étant des lysosomes [26].

On peut aussi observer ces lésions dans les cas de néphropathies liées à des produits avec un haut pouvoir osmotique comme les produits de contraste iodés [41], les produits de remplissage vasculaire [42] (hydroxyéthylamidons en particulier) ou le mannitol.

Les préparations d'IgIV à base de saccharose ont un pouvoir osmotique supérieur aux autres préparations (glucose, maltose, acides aminés).

Certains expliquent la meilleure tolérance du maltose par la présence de la maltase qui dégrade le maltose en glucose sur la bordure en brosse des cellules tubulaires proximales. En revanche, il n'existe pas d'enzyme dégradant le saccharose dans ces mêmes cellules. Dans l'étude de la base de données de la FDA, on note que les facteurs de risque de survenue de l'IRA sont le saccharose (90\% des patients), l'âge supérieur à 65 ans $(65 \%)$, le diabète ( $56 \%$ ) et l'insuffisance rénale préexistante (26\%) [37].

Devant l'augmentation des cas d'IRA, l'Afssaps a publié en novembre 2006 un communiqué déconseillant les IgIV contenant du saccharose pour ces patients à risque. Elle reprenait les facteurs de risque de la FDA en rajoutant les patients hypovolémiques et obèses (Tableau 2). En effet, chez les patients obèses, le dosage est souvent calculé par rapport au poids mesuré et non pas au poids idéal. Cette mesure surestime le volume plasmatique et conduit à une augmentation des effets indésirables des IgIV. Chez ces patients à risque, l'usage des IgIV impose par ailleurs une hydratation correcte orale ou intraveineuse avant l'administration, de surveiller la diurèse, de doser la créatininémie avant et après la cure idéalement et d'éviter d'associer des diurétiques de l'anse. Un algorithme décisionnel en cas de prescription d'IgIV en réanimation est proposé en figure 3.

Même si les préparations à base de saccharose sont évitées chez les patients à risque et en particulier en néphrologie, les autres IgIV ne sont pas dépourvues de néphrotoxicité. En effet, des cas d'IRA avec des préparations contenant du maltose ont été décrits en 2006 [43]. À partir de 2010, l'utilisation des IgIV sans saccharose a été répandue en vue

Tableau 2 Facteurs de risque de néphrotoxicité des IgIV

Saccharose comme excipient Obésité

Diabète

Hypovolémie

Insuffisance rénale chronique Autres excipients glucidiques

Âge $>65$ ans

Âge du donneur

en transplantation rénale 


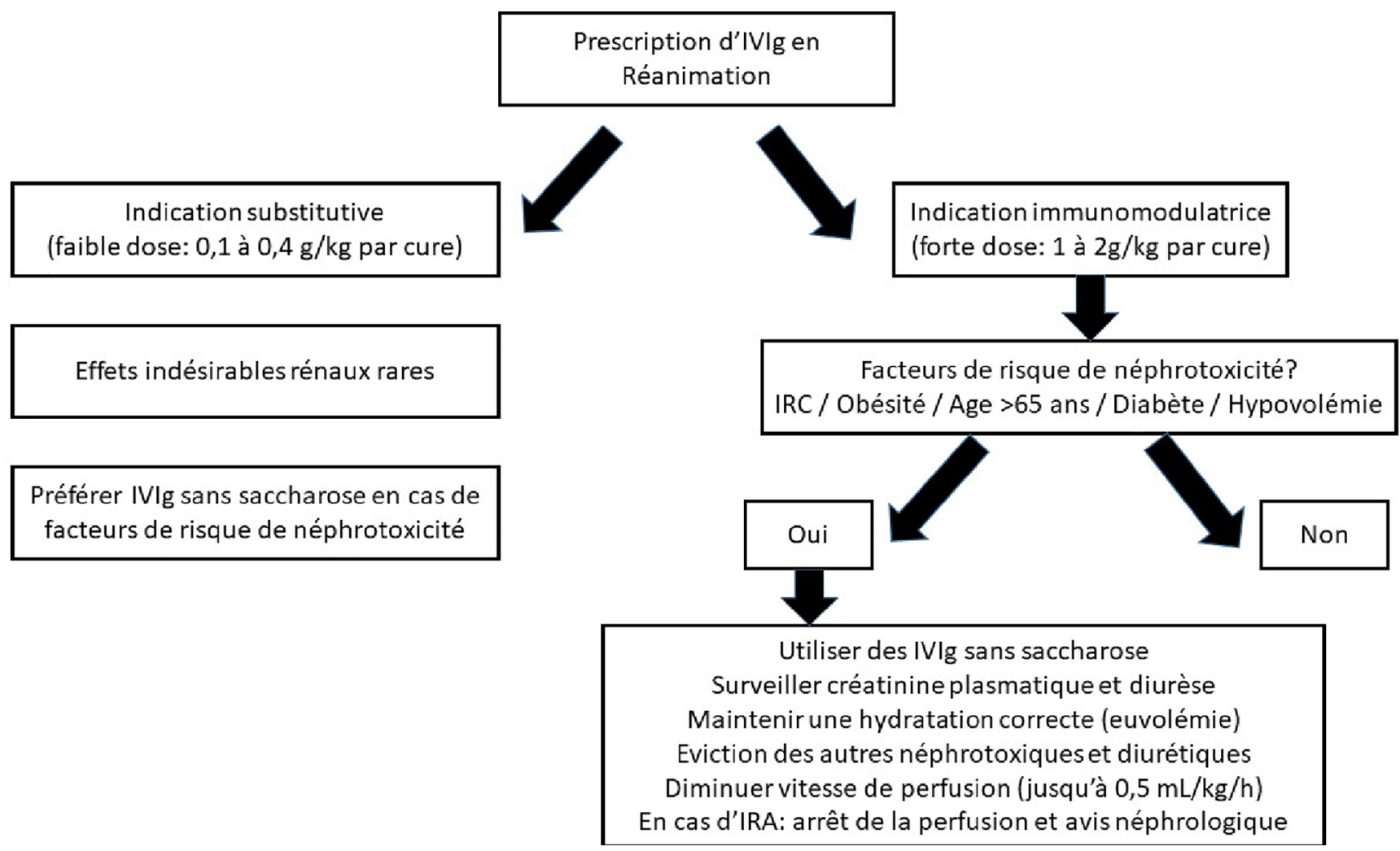

Fig. 3 Algorithme décisionnel pour la prescription d'IgIV en réanimation en fonction des facteurs de risque de néphrotoxicité

de la diminution du risque d'IRA avec ces nouveaux produits [44]. Deux études récentes se sont intéressées à la toxicité rénale des IgIV chez les transplantés rénaux où ces produits sont largement utilisés depuis le début des années 2000 dans le but de contrôler l'immunisation anti-HLA et le risque de rejet humoral $[45,46]$. La perfusion d'IgIV sans saccharose, dans ces deux études, ne s'accompagne pas de cas d'IRA chez des patients transplantés rénaux récents. Cependant, l'analyse des biopsies protocolaires du greffon rénal révèle des lésions tubulaires dans un grand nombre de cas. Celles-ci sont associées à une fibrose interstitielle et à une moins bonne fonction rénale à un an dans une des études [45]. La qualité du greffon (âge du donneur et fibrose interstitielle initiale) semble être un des facteurs favorisant la susceptibilité rénale aux IgIV $[45,46]$. La seconde étude suggère que les IgIV sans excipient glucidique seraient moins associées à ces lésions tubulaires [46].

Il est à noter que l'on manque de données pour évaluer la toxicité tubulaire des perfusions d'IgIV au long cours et leurs conséquences sur la fibrogenèse et le déclin de la fonction rénale. Une étude rétrospective récente et non contrôlée décrit une baisse du débit de filtration glomérulaire estimé à plus de $20 \%$ chez $10 \%$ des 166 patients traités pendant un an par IgIV [47]. D'autres études sont indispensables pour mieux décrire la toxicité rénale chronique de ces produits qui est suggérée par exemple par le fait que les vacuoles tubulaires liées aux perfusions d'IgIV réalisées en post-transplantation précoce peuvent persister à distance de la greffe.

\section{Conclusion}

Les IgIV sont des spécialités coûteuses et largement utilisées de nos jours. Les indications continuent d'augmenter surtout à visée immunomodulatrice où les doses utilisées sont élevées. La néphrotoxicité de ces produits est tubulaire et est principalement associée aux formulations contenant du saccharose. Les cas d'IRA sont exceptionnels avec les formulations sans cet excipient glucidique. Cependant, on note aussi des lésions histologiques tubulaires infracliniques avec les autres formulations, et la toxicité rénale des perfusions au long cours reste inconnue. Les IgIV avec saccharose doivent donc être évitées chez les patients à risque d'insuffisance rénale qui sont les patients âgés, diabétiques, obèses, en hypovolémie, transplantés rénaux ou ayant une insuffisance rénale chronique sous-jacente. Des études supplémentaires sont nécessaires pour évaluer la toxicité chronique de ces produits utilisés à grande échelle et parfois pendant de longues durées.

Liens d'intérêts : l'auteur déclare ne pas avoir de lien d'intérêt. 


\section{Références}

1. Nimmerjahn F, Ravetch JV, (2008) Anti-inflammatory actions of intravenous immunoglobulin. Annu Rev Immunol 26: 513-533. doi: 10.1146/annurev.immunol.26.021607.090232

2. Imbach P, Barandun S, d'Apuzzo V, Baumgartner C, Hirt A, Morell A, Rossi E, Schöni M, Vest M, Wagner HP, (1981) High-dose intravenous gammaglobulin for idiopathic thrombocytopenic purpura in childhood. Lancet 1: 1228-1231

3. Looney RJ, Huggins J, (2006) Use of intravenous immunoglobulin G (IVIG). Best Pract Res Clin Haematol 19: 3-25. doi: 10.1016/j.beha.2005.01.032

4. Kazatchkine MD, Kaveri SV, (2001) Immunomodulation of autoimmune and inflammatory diseases with intravenous immune globulin. $\mathrm{N}$ Engl J Med 345: 747-755. doi: 10.1056/ NEJMra993360

5. Durandy A, Kaveri SV, Kuijpers TW, Basta M, Miescher S, Ravetch JV, Rieben R, (2009) Intravenous immunoglobulins - understanding properties and mechanisms. Clin Exp Immunol 158: 2-13. doi: 10.1111/j.1365-2249.2009.04022.x

6. Durandy A, Wahn V, Petteway S, Gelfand EW, (2005) Immunoglobulin replacement therapy in primary antibody deficiency diseases--maximizing success. Int Arch Allergy Immunol 136: 217-229. doi: 10.1159/000083948

7. Molica S, Musto P, Chiurazzi F, Specchia G, Brugiatelli M, Cicoira L, Levato D, Nobile F, Carotenuto M, Liso V, Rotoli B, (1996) Prophylaxis against infections with low-dose intravenous immunoglobulins, (IVIG) in chronic lymphocytic leukemia. Results of a crossover study. Haematologica 81: 121-126

8. Sullivan KM, Kopecky KJ, Jocom J, Fisher L, Buckner CD, Meyers JD, Counts GW, Bowden RA, Peterson FB, Witherspoon RP, Budinger MD, Schwartz RS, Appelbaum FR, Clift RA, Hansen JA, Sanders JE, Thomas ED, Storb , (1990) Immunomodulatory and antimicrobial efficacy of intravenous immunoglobulin in bone marrow transplantation. $\mathrm{N}$ Engl J Med 323: 705-712. doi: 10.1056/NEJM199009133231103

9. Schwab I, Nimmerjahn F, (2013) Intravenous immunoglobulin therapy: how does IgG modulate the immune system? Nat Rev Immunol 13: 176-189. doi: 10.1038/nri3401

10. Le Pottier L, Sapir T, Bendaoud B, Youinou P, Shoenfeld Y, Pers $\mathrm{JO}$, (2007) Intravenous immunoglobulin and cytokines: focus on tumor necrosis factor family members BAFF and APRIL. Ann N Y Acad Sci 1110: 426-432. doi: 10.1196/annals.1423.044

11. Prasad NK, Papoff G, Zeuner A, Bonnin E, Kazatchkine MD, Ruberti G, Kaveri SV, (1998) Therapeutic preparations of normal polyspecific IgG (IVIg) induce apoptosis in human lymphocytes and monocytes: a novel mechanism of action of IVIg involving the Fas apoptotic pathway. J Immunol 161: 3781-3790

12. Viard I, Wehrli P, Bullani R, Schneider P, Holler N, Salomon D, Hunziker T, Saurat JH, Tschopp J, French LE, (1998) Inhibition of toxic epidermal necrolysis by blockade of CD95 with human intravenous immunoglobulin. Science 282: 490-493

13. Debré M, Bonnet MC, Fridman WH, Carosella E, Philippe N, Reinert P, Vilmer E, Kaplan C, Teillaud JL, Griscelli C, (1993) Infusion of $\mathrm{Fc}$ gamma fragments for treatment of children with acute immune thrombocytopenic purpura. Lancet 342: 945-949

14. Samuelsson A, Towers TL, Ravetch JV, (2001) Anti-inflammatory activity of IVIG mediated through the inhibitory Fc receptor. Science 291: 484-486. doi: 10.1126/science.291.5503.484

15. Tackenberg B, Jelcic I, Baerenwaldt A, Oertel WH, Sommer N, Nimmerjahn F, Lünemann JD, (2009) Impaired inhibitory Fcgamma receptor IIB expression on B cells in chronic inflammatory demyelinating polyneuropathy. Proc Natl Acad Sci U S A 106: 4788-4792. doi: 10.1073/pnas.0807319106
16. Ephrem A, Chamat S, Miquel C, Fisson S, Mouthon L, Caligiuri G, Delignat S, Elluru S, Bayry J, Lacroix-Desmazes S, Cohen JL, Salomon BL, Kazatchkine MD, Kaveri SV, Misra $\mathrm{N}$, (2008) Expansion of CD4+CD25+ regulatory $\mathrm{T}$ cells by intravenous immunoglobulin: a critical factor in controlling experimental autoimmune encephalomyelitis. Blood 111: 715722. doi: 10.1182/blood-2007-03-079947

17. Séité JF, Cornec D, Renaudineau Y, Youinou P, Mageed RA, Hillion S, (2010) IVIg modulates BCR signaling through CD22 and promotes apoptosis in mature human B lymphocytes. Blood 116: 1698-1704. doi: 10.1182/blood-2009-12-261461

18. Kaneko Y, Nimmerjahn F, Ravetch JV, (2006) Anti-inflammatory activity of immunoglobulin $\mathrm{G}$ resulting from $\mathrm{Fc}$ sialylation. Science 313: 670-673. doi: 10.1126/science.1129594

19. Schwab I, Biburger M, Krönke G, Schett G, Nimmerjahn F, (2012) IVIg-mediated amelioration of ITP in mice is dependent on sialic acid and SIGNR1. Eur J Immunol 42: 826-830. doi: 10.1002/eji.201142260

20. Burks AW, Sampson HA, Buckley RH, (1986) Anaphylactic reactions after gamma globulin administration in patients with hypogammaglobulinemia. Detection of IgE antibodies to IgA. N Engl J Med 314: 560-564. doi: 10.1056/NEJM198602273140907

21. Welles CC, Tambra S, Lafayette RA, (2010) Hemoglobinuria and acute kidney injury requiring hemodialysis following intravenous immunoglobulin infusion. Am J Kidney Dis 55: 148-151. doi: 10.1053/j.ajkd.2009.06.013

22. Matsuda M, Hosoda W, Sekijima Y, Hoshi K, Hashimoto T, Itoh S, Ikeda S, (2003) Neutropenia as a complication of high-dose intravenous immunoglobulin therapy in adult patients with neuroimmunologic disorders. Clin Neuropharmacol 26: 306-311

23. Reinhart WH, Berchtold PE, (1992) Effect of high-dose intravenous immunoglobulin therapy on blood rheology. Lancet 339: 662-664

24. Go RS, Call TG, (2000) Deep venous thrombosis of the arm after intravenous immunoglobulin infusion: case report and literature review of intravenous immunoglobulin-related thrombotic complications. Mayo Clin Proc Mayo Clin 75: 83-85. doi: 10.4065/75.1.83

25. Katz KA, Hivnor CM, Geist DE, Shapiro M, Ming ME, Werth VP, (2003) Stroke and deep venous thrombosis complicating intravenous immunoglobulin infusions. Arch Dermatol 139: 991-993. doi: 10.1001/archderm.139.8.991

26. Dickenmann M, Oettl T, Mihatsch MJ, (2008) Osmotic nephrosis: acute kidney injury with accumulation of proximal tubular lysosomes due to administration of exogenous solutes. Am J Kidney Dis 51: 491-503. doi: 10.1053/j.ajkd.2007.10.044

27. Barton JC, Herrera GA, Galla JH, Bertoli LF, Work J, Koopman WJ, (1987) Acute cryoglobulinemic renal failure after intravenous infusion of gamma globulin. Am J Med 82: 624-629

28. Ruggeri M, Castaman G, De Nardi G, Rodeghiero F, (1993) Acute renal failure after high-dose intravenous immune globulin in a patient with idiopathic thrombocytopenic purpura. Haematologica 78: 338-339

29. Cayco AV, Perazella MA, Hayslett JP, (1997) Renal insufficiency after intravenous immune globulin therapy: a report of two cases and an analysis of the literature. J Am Soc Nephrol 8: 1788-1794

30. Decocq G, de Cagny B, Andréjak M, Desablens B, (1996) Acute kidney failure secondary to intravenous immunoglobulin administration. 4 cases and review of the literature. Therapie 51: 516-526

31. Arunabh S, Kumar G, Avila V, (1996) Acute renal failure induced by intravenous immune globulin. Am Fam Physician 53: 862-865

32. Ahsan N, Wiegand LA, Abendroth CS, Manning EC, (1996) Acute renal failure following immunoglobulin therapy. Am J Nephrol 16: 532-536 
33. Sati HI, Ahya R, Watson HG, (2001) Incidence and associations of acute renal failure complicating high-dose intravenous immunoglobulin therapy. Br J Haematol 113: 556-557

34. Fakhouri F, (2007) Intravenous immunoglobulins and acute renal failure: mechanism and prevention. Rev Med Interne 28: 4-6

35. Dantal J, (2013) Intravenous immunoglobulins: in-depth review of excipients and acute kidney injury risk. Am J Nephrol 38: 275-284. doi: 10.1159/000354893

36. Brannagan TH 3rd, Nagle KJ, Lange DJ, Rowland LP, (1996) Complications of intravenous immune globulin treatment in neurologic disease. Neurology 47: 674-677

37. Centers for Disease Control and Prevention (CDC), (1999) Renal insufficiency and failure associated with immune globulin intravenous therapy — United States, 1985-1998. MMWR Morb Mortal Wkly Rep 48: 518-521

38. Perazella MA, Cayco AV, (1998) Acute renal failure and intravenous immune globulin: sucrose nephropathy in disguise? Am J Ther 5: 399-403

39. Zhang R, Szerlip HM, (2000) Reemergence of sucrose nephropathy: acute renal failure caused by high-dose intravenous immune globulin therapy. South Med J 93: 901-904

40. Khalil M, Shin HJ, Tan A, DuBose TD Jr, Ordóñez N, Katz RL, (2000) Macrophagelike vacuolated renal tubular cells in the urine of a male with osmotic nephrosis associated with intravenous immunoglobulin therapy. A case report. Acta Cytol 44: 86-90

41. Moreau JF, Noel LH, Droz D, (1993) Proximal renal tubular vacuolization induced by iodinated contrast media, or so-called "osmotic nephrosis." Invest Radiol 28: 187-190
42. Legendre C, Thervet E, Page B, Percheron A, Noël LH, Kreis H, (1993) Hydroxyethylstarch and osmotic-nephrosis-like lesions in kidney transplantation. Lancet 342: 248-249

43. Chacko B, John GT, Balakrishnan N, Kirubakaran MG, Jacob CK, (2006) Osmotic nephropathy resulting from maltose-based intravenous immunoglobulin therapy. Ren Fail 28: 193-195

44. Chapman SA, Gilkerson KL, Davin TD, Pritzker MR, (2004) Acute renal failure and intravenous immune globulin: occurs with sucrose-stabilized, but not with D-sorbitol-stabilized, formulation. Ann Pharmacother 38: 2059-2067. doi: 10.1345/aph.1E040

45. Bollée G, Anglicheau D, Loupy A, Zuber J, Patey N, Mac Gregor D, Martinez F, Mamzer-Bruneel MF, Snanoudj R, Thervet E, Legendre C, Noël LH, (2008) High-dosage intravenous immunoglobulin-associated macrovacuoles are associated with chronic tubulointerstitial lesion worsening in renal transplant recipients. Clin J Am Soc Nephrol 3: 1461-1468. doi: 10.2215/CJN.00500108

46. Luque Y, Anglicheau D, Rabant M, El Karoui K, Jamme M, Aubert O, Clément R, Noël LH, Bollée G, Brodin-Sartorius A, Martinie M, Kreis H, Timsit MO, Legendre C, (2016) Renal safety of high-dose, sucrose-free intravenous immunoglobulin in kidney transplant recipients: an observational study. Transpl Int 29: 1205-1215 doi: 10.1111/tri.12833

47. Levine AA, Levine TD, Clarke K, Saperstein D, (2017) Renal and hematologic side effects of long-term intravenous immunoglobulin therapy in patients with neurologic disorders. Muscle Nerve 56: 1173-1176. doi: 10.1002/mus.25693 